\title{
La loi de 1912 sur la circulation des « nomades » (Tsiganes) en France
}

\section{Emmanuel Filhol}

\section{(2) OpenEdition \\ 1 Journals}

\section{Édition électronique}

URL : https://journals.openedition.org/remi/4179

DOI : $10.4000 /$ remi.4179

ISSN : $1777-5418$

Éditeur

Université de Poitiers

\section{Édition imprimée}

Date de publication : 1 octobre 2007

Pagination : 135-158

ISBN : 978-2-911627-46-6

ISSN : 0765-0752

\section{Référence électronique}

Emmanuel Filhol, "La loi de 1912 sur la circulation des « nomades » (Tsiganes) en France », Revue européenne des migrations internationales [En ligne], vol. 23 - n² | 2007, mis en ligne le 01 octobre 2010, consulté le 14 avril 2022. URL : http://journals.openedition.org/remi/4179 ; DOI : https://doi.org/ 10.4000/remi.4179

Ce document a été généré automatiquement le 14 avril 2022.

(c) Université de Poitiers 


\title{
La loi de 1912 sur la circulation des « nomades » (Tsiganes) en France
}

\author{
Emmanuel Filhol
}

1 L'enracinement tsigane est attesté en France depuis le $\mathrm{XV}^{\mathrm{e}}$ siècle ${ }^{1}$. Contrairement à une image répandue, les Tsiganes ont été au début bien accueillis. Ils bénéficièrent dans ce pays et partout ailleurs en Europe de l'hospitalité auprès des autorités municipales et des nobles, des bonnes grâces accordées par les cours royales et princières, une attitude bienveillante qui prévalut à leur égard jusqu'au XVII ${ }^{\mathrm{e}}$ siècle ${ }^{2}$. Mais à partir des années 1600 , surtout de la deuxième moitié du XVII ${ }^{\mathrm{e}}$ siècle, le destin de la diffuse «nation bohémienne " bascule, on assiste à une montée des comportements hostiles et, avec elle, à l'établissement rigoureux et constant de sanctions effectives ${ }^{3}$. La sévérité des textes est exemplaire dans sa cohérence ; elle est générale, et pas seulement française (H. Grellmann, 1810 ; p. Bataillard, 1843-1844 ; F. de Vaux de Foletier, 1970 : 75-89 ; H. Asséo, 1974 ; B. Leblon, 1985 ; A. Fraser, 1992 ; E. Filhol, 2002). Privés de l'accueil des châteaux, où les Égyptiennes et leurs spectacles de danse suscitaient un véritable engouement, exclus des compagnies de gens d'armes, chassés des villes, la situation des Tsiganes se dégrade rapidement; ceux-ci sont confondus avec les «errants et vagabonds » et pourchassés à ce titre dans toute l'Europe. Les interdictions de contacts et l'impossibilité d'exercer une activité itinérante mais légale condamnent le peuple bohême au bannissement collectif. La déclaration de juillet 1682, voulue par Colbert et signée de Louis XIV, "contre les Bohèmes et ceux qui leur donnent retraite " (Isambert, Decrusy, Taillandier, 1821-1833, t. XIX : 393-394), demande aux baillis, sénéchaux et leurs lieutenants d'arrêter et de faire arrêter les hommes afin qu'on les conduise aux galères, à perpétuité, en dehors de tout délit constaté, tandis que les femmes, séparées des premiers, seront tondues et au cas où elles continueraient à "mener la vie de bohémiennes", passibles d'être fustigées et bannies; quant aux enfants, et c'est la première fois qu'un texte prévoit leur sort, ils seront conduits dans les hôpitaux pour y être élevés selon la religion chrétienne. Au siècle suivant, le traitement administratif des Bohémiens est englobé dans celui des vagabonds, mendiants et gens sans aveu. Les innombrables textes consacrés à la condamnation du 
vagabondage et la réprobation de la mendicité soumettent les compagnies tsiganes aux exigences de domiciliation et au contrôle.

Le destin des Tsiganes bascula une fois de plus vers la fin du XIX ${ }^{e}$ siècle, quand de nouvelles migrations de Tsiganes venus d'Europe danubienne et balkanique furent provoquées par la libération des liens de servage. L'arrivée de ces petits groupes familiaux singulièrement exotiques déclencha une vague d'hostilité, et l'on accusa les Bohémiens, après la guerre de 1870, d'être des agents étrangers au service de l'Allemagne. Les mesures qui suivirent, fondées sur un renforcement des pratiques de surveillance, d'identification et de contrôle vis-à-vis des Tsiganes voyageurs, aboutiront à la promulgation de la loi de 1912 relative à la circulation des "nomades", un dispositif législatif particulièrement discriminatoire et vexatoire.

\section{La situation à la fin du $\mathrm{XIX}^{\mathrm{e}}$ siècle}

3 En 1895, un dénombrement général de tous les «nomades, bohémiens, vagabonds » est prescrit par le gouvernement. Seules les archives de vingt-deux départements ont conservé les résultats de l'enquête. Ainsi le recensement du 20 mars effectué par les brigades de gendarmerie et les gardes champêtres dans le département de la CharenteInférieure contient des informations importantes sur les Tsiganes circulant en Saintonge: il indique les noms et prénoms des membres de la «bande», leur " profession apparente », leur nationalité, leur sexe et âge, leur lieu de naissance, le lieu d'où ils viennent, le lieu où ils disent se rendre, les papiers dont ils sont porteurs, les dispositions particulières prises par les maires à l'égard des bandes et des individus isolés, des «observations". L'enquête recense 585 itinérants considérés comme nomades, dont les métiers déclarés sont saltimbanque, vannier, chanteur ambulant, marchand forain, lutteur, colporteur, acrobate, écuyer de cirque, graveur, boulanger, artiste d'agilité, marchand d'oiseaux, fabricant de corbeilles, marchand de chevaux, fabricant de cadres de coquillages... Quelques familles paraissent assez étoffées : les Patrac regroupent une trentaine de personnes; marchands de chevaux, ce sont des Gitans originaires des départements pyrénéens et de la région de la Garonne. Sur toutes les listes établies pour le recensement en Charente-Inférieure, on remarque une forte proportion de familles de voyageurs, d'origine sinté ou manouche (le mot signifie "homme» dans la langue tsigane), plusieurs d'entre elles munies de certificats d'option après avoir quitté les départements d'Alsace-Lorraine, annexés par la Prusse en 1871. Parmi les noms relevés, citons divers patronymes français : Durosier, Hulot, Moisdon, Bertaux, Chevalier, Thomas, Lafont, Flore, Larose, Lemerle, Pain, Renard, Pentecôte, Lespinasse, André, Martin. Les Tsiganes qui se déplacent en Saintonge sont majoritairement de nationalité française ${ }^{4}$. L'enquête mentionne également des Italiens, Autrichiens, Espagnols, mais aucune famille venant d'Europe centrale ou orientale. D'après les renseignements indiqués par les gendarmes dans la rubrique " observations ", les moyens de transport qu'utilisent les nomades charentais sont des roulottes de bois. Soit, comme pour d'autres familles tsiganes recensées ailleurs, de vraies maisons roulantes, complètement fermées, avec des portes, des fenêtres, un poêle dont le tuyau traverse sur un côté le toit bombé. Soit, le plus souvent, des voitures bâchées ("voiture à bras, couverte d'une toile noire goudronnée »), dont la caisse est peinte en bleu, ou en vert, ou en d'autres couleurs. Voitures à deux roues, attelées à un seul cheval, à un âne ou à un mulet (E. Filhol, 2003 : 53-55). 
Dans le département des Landes, le recensement des nomades, si l'on considère l'ensemble des réactions transmises par les mairies, donna lieu de la part des communes à une mobilisation effective, les autorités municipales sollicitant même parfois la participation de certains habitants, comme l'atteste cette déclaration du maire de Tarnos: "J'ai chargé spécialement des habitants de la surveillance de nos deux routes nationales $n^{\circ} 10$ et 117 et de nos principaux chemins vicinaux et de prendre les noms des nomades et bohémiens qui s'y montreraient ou qui stationneraient. Au besoin, ils me sont amenés. En outre, je me suis concerté avec la gendarmerie voisine ». La commune de Saugnac-et-Cambran s'assura quant à elle de «l'aide d'autres personnes de bonne volonté postées dans les principaux quartiers et hameaux de la commune ». Mentionnons aussi le fait que diverses localités, ayant déclaré ne pas avoir vu ce jour-là passer des nomades, soit parce que, selon les dires du sous-préfet de Saint-Sever, « les bandes de bohémiens de passage dans les localités sont presque toujours expulsées du territoire où elles ont stationné, le jour même de leur arrivée ", ou parce que les familles préfèrent se diriger vers des communes voisines plus importantes, se diront prêtes à reconduire l'opération une autre fois. Le maire de Mimbaste écrit au sous-préfet de Dax: "J'aurai soin, si l'occasion se présente, de surveiller avec attention les nomades en question, de visiter les papiers dont ils sont porteurs, et de vous signaler leurs agissements ». La municipalité de Parleboscq déclare apporter son soutien pour aider l'administration «à réprimer ce dangereux vagabondage qui s'étend partout, et généralement, n'est que trop toléré ». Cette commune signale par ailleurs qu'il y a environ un mois et demi est venue « une bande de bohémiens hongrois ou autres, qui a séjourné 48 heures, hommes, femmes, enfants, équipages, chevaux, campements, animaux ». La conclusion du maire est catégorique : " Les vauriens de cette espèce devraient être expulsés sans trêve ni merci ». Le maire de Vicq-d'Auribat assure de son côté qu'il relèvera toutes les indications nécessaires s'il survient ultérieurement des nomades et bohémiens, ajoutant que "s'il y avait résistance de leur part, la gendarmerie serait immédiatement prévenue ». À l'occasion du recensement, le ministre de l'Intérieur ordonna une enquête sur un problème qui le préoccupait. Il télégraphia aux préfets : «Suis informé que bandes nomades parcourent France et étranger, obéissant à un chef résidant à Paris. Il y a intérêt capital à découvrir ce chef et à connaître la nature des liens qui l'unissent à nomades, des ordres qu'il leur donne et des missions qu'il leur confie ». Compte tenu, suite à la défaite de 1871, de l'exacerbation du patriotisme français vis-à-vis de l'Allemagne, il n'est guère surprenant que des bruits aient couru à propos d'un prétendu « chef » tsigane venu de l'étranger, perçu sans doute comme un espion au service de l'ennemi. Des noms circulèrent alors, parmi lesquels celui du nomade Marc Eugène : «me faire connaître d'urgence si lors du recensement des nomades effectué le 20 mars dernier la gendarmerie a relevé le nom d'un nommé Marc Eugène né au Havre le 7 novembre 1877 ayant exercé la profession de Boucher à Caumont Calvados et étant parti de cette localité en juillet dernier pour s'engager semblerait-il dans un cirque $»^{5}$. Les chiffres du recensement disponibles aux Archives départementales des Landes ne recouvrent pas la totalité des communes du département mais seulement trente-neuf d'entre elles. On y dénombre 216 nomades. Leurs métiers sont rétameur, saltimbanque, colporteur de livres et papiers à lettres, vannier, siffleur, chanteur ou musicien ambulant, marchand forain, marchand d'aiguilles et vendeur de chaussons, marchand de paniers, tondeur de chevaux, marchand d'ânes, lutteur, boulanger, ouvrier, mendiant, garçon d'hôtel, comédien, acrobate, écuyer de cirque, directeur de théâtre, graveur, boulanger, artiste 
d'agilité, artiste lyrique, laboureur, maquignon, fabricant de tabourets, terrassier, tapissier, raccommodeur de parapluies, tailleur... Plusieurs familles se composent au moins d'une vingtaine de personnes ; les Lambert, par exemple, exerçant la profession de marchand de chevaux, saltimbanque, sont des Tsiganes originaires de Suisse, des départements de l'Isère et des Côtes-du-Nord. Les listes établies pour le recensement des Bohémiens des Landes révèlent une forte proportion de familles de voyageurs, presque toutes manouches, dont certaines provenant également d'Alsace et de Moselle avaient opté pour la France en 1872, après l'annexion par l'Allemagne, et des familles gitanes. La plupart portent des noms français : Barrez, Valin, Laurent, Ramelot, Laffon, Rosier, Bertrand, Gastaud, Lafitte, Vignau, Duran, Barate, Boutaloup, Conche, Ornecq, Laffleur, Bellocq, Châtelain, Lacroix, Sabatier, Lunel, Ory, Rivière, Antoine. Les Tsiganes qui se déplacent dans le département sont majoritairement de nationalité française. L'enquête mentionne aussi des Espagnols, Italiens, Suisses. Précisons que si le recensement avait été fait peu de temps avant ou postérieurement à la date retenue, d'autres familles originaires d'Europe centrale et balkanique, des Roms (littéralement les «hommes": nom donné à un autre groupe tsigane) hongrois et bosniaques en particulier, auraient pu figurer sur les listes. Un rapport de gendarmerie, daté du 2 septembre 1895, nous apprend en effet que les familles de Victor Stancovitch et Costa Stéfanovitch, nés en Bosnie, "conduisant deux ours et deux singes ", porteurs de passeports délivrés par le consul de l'Empire Ottoman, stationnèrent à ce moment dans la rue principale de Gabarret, avant de repartir le lendemain matin en direction du Gard, lieu de leur destination. En ce qui concerne les papiers administratifs présentés par les nomades recensés, il s'agit surtout de livret individuel, de livret de famille (l'un des livrets comporte la notation suivante: "Cet individu est estropié de la jambe gauche. Il est accompagné de sa femme Marie et de leur fille âgée de 12 ans. Atteint de myopie. Bien mis, figure sympathique »), de carnet professionnel, de livret d'ouvrier, de livret militaire, ou encore de passeport intérieur, de certificat de travail, de certificats délivrés par les maires des communes traversées, voire de certificat de maladie. Un Tsigane voyageant seul près de Montfort, Eugène Roban, âgé de 52 ans, possède un vieux livret d'ouvrier et un livret de la Caisse nationale d'épargne ${ }^{6}$.

5 Le recensement des «nomades en bande voyageant en roulotte", dont le nombre sera estimé à 25 000, rencontra dans la presse populaire un écho largement favorable : « Le ministre de l'intérieur a voulu régulariser, autant que possible, la situation de ces errants au milieu desquels peuvent se cacher nos pires ennemis. Il a ordonné leur recensement général. Le même jour, à la même heure, partout en France, ils ont été cernés par la gendarmerie ; il leur a fallu dire leurs noms, prénoms et lieux d'origine, de sorte que maintenant il sera possible de les soumettre aux lois qui régissent les étrangers en France $»^{7}$.

\section{Les étapes préparatoires à la loi}

6 À partir de 1897, toute une série de propositions parlementaires concernant d'abord les mendiants, vagabonds et nomades, puis le traitement des professions ambulantes et des nomades, vise à surveiller et marginaliser les Tsiganes. Après avoir préconisé, dans le cadre d'un renforcement de la police rurale et municipale, l'obligation pour tout nomade d'être pourvu d'une "feuille d'identité qui comprendrait, en outre du signalement et de la profession, la photographie de l'intéressé. Cet usage [...] aurait un 
précieux avantage s'il était exigé des nomades qui font souvent usage de faux papiers et de faux noms $"^{8}$, le sénateur Louis Émile de Marcère, républicain de centre gauche, président de la commission extra-parlementaire instituée le 13 novembre 1897 et chargée d'améliorer la police du vagabondage, propose au gouvernement un projet d'arrêté préfectoral dont la mesure principale est d'exiger comme condition préalable à la circulation des familles bohémiennes et à l'exercice de leur profession ambulante l'obtention d'une autorisation préfectorale. Celle-ci ne pourra être délivrée que sur présentation d'une pièce d'identité, soit par un passeport ou un livret d'ouvrier, soit par une carte d'identité individuelle ou collective contenant l'état civil, le signalement exact et l'indication du dernier domicile ou de la résidence de celui qui en sera détenteur, carte d'identité mise à la disposition des intéressés, dans les bureaux des préfectures et sous-préfectures. À défaut de ces papiers, l'article 3 prévoit que «tout individu visé par le présent arrêté, qui ne serait pas en mesure d'établir son identité par l'une des pièces énumérées, sera retenu administrativement à l'effet de rechercher cette identité. Pour le cas où ces recherches resteraient infructueuses, il sera déféré aux tribunaux sous l'inculpation de vagabondage ${ }^{9}$. Bien que le ministre Louis Barthou, tout en adoptant les vues de la commission, jugeât qu'une mesure aussi grave que l'obligation d'une carte d'identité ne pouvait être imposée par un arrêté réglementaire, puisque depuis la suppression des passeports intérieurs, nul n'était tenu, à moins d'être incriminé de vagabondage (défini d'après les critères du défaut de domicile, de l'absence de travail régulier et de moyens d'existence), d'établir son identité et d'être porteur de papiers comme le passeport ou le livret d'ouvrier supprimés, l'un et l'autre, les recommandations émises par la commission ne sont pas pour autant négligées. Au cours des deux années suivantes, plusieurs préfets s'en inspirent, ceux des départements du Pas-de-Calais, de la Vienne et de la Haute-Vienne, prenant des arrêtés qui imposent aux maires de surveiller les nomades en roulotte jusqu'à leur sortie du territoire communal et soumettent les Bohémiens à l'obligation de se munir d'une double autorisation du préfet et du maire pour pouvoir stationner. Le rôle actif joué par les conseils généraux dans ces décisions préfectorales mérite aussi d'être souligné. Ainsi, en Gironde, lors de sa séance du 23 avril 1903, le conseil général, en la personne du conseiller Édouard Bertin, avocat à la Cour de Bordeaux, vice-président de la Ligue de l'Enseignement (en 1900), et rapporteur de la commission relative à la "répression du vagabondage et de la mendicité dans les campagnes ", demande au nouveau préfet Charles Lutaud de mettre en application les mesures de répression déjà en vigueur dans les départements précités, comme le suggère par ailleurs la puissante Société des agriculteurs de France au président du conseil général, « en attendant que le Parlement fasse sortir de ses cartons le projet de loi auquel fait allusion la délibération des agriculteurs de France $»^{10}$. Un arrêté préfectoral particulièrement coercitif voit le jour le 20 octobre 1904, selon lequel les nomades chanteurs ou musiciens ambulants (et tout musicien, saltimbanque, prestidigitateur, directeur de cirque, tirs, jeux d'adresse, etc.) résidant en Gironde ne peuvent exercer leur activité que s'ils possèdent une autorisation préfectorale, à condition de fournir « l'avis du Maire du domicile, appuyé d'un certificat de moralité délivré par celui-ci ou par le Commissaire de police et d'un signalement détaillé ». Quant aux "nomades indigents », non pourvus de papiers, la préfecture a prévu de procéder à leur immatriculation en les dotant d'un «carnet modèle ", avec feuilles détachables, visé par les mairies, qui renferme outre le signalement, des renseignements sur chaque passage du nomade dans une commune, la date et le lieu où il a passé la nuit et l'endroit où il compte se rendre pour dormir le 
lendemain, car ce dispositif constitue «l'un des moyens les plus efficaces de suivre et de retrouver, s'il en était besoin, la trace des nomades à travers le département ». Le but recherché est on ne peut plus clair, il s'agit de «débarrasser le département, les campagnes principalement, de cette population nomade composée en partie de gens sans profession avouée, le plus souvent dangereux ». D'où la mise en place d'un véritable cordon sanitaire imaginé par l'autorité préfectorale pour dissuader les vagabonds et nomades de pénétrer en Gironde : le préfet convie en effet les maires, qui « recevront, en placard, l'arrêté qu'ils voudront bien faire immédiatement publier et afficher dans leurs communes respectives ", à placarder cet arrêté dans les communes limitrophes, «notamment aux abords des routes donnant accès dans le département $\aleph^{11}$. Mais ce n'est pas tout. L'arrêté du préfet Lutaud prévoyait initialement que les nomades musiciens ou chanteurs ambulants en provenance des autres départements devaient justifier de leur identité et eux aussi être porteurs d'une autorisation délivrée par la préfecture de la Gironde. Le ministre de l'Intérieur et président du conseil Émile Combes ayant considéré ces prescriptions excessives (en raison du même argument que celui invoqué par ses prédécesseurs Barthou et Waldeck-Rousseau, à propos de la première ; en ce qui concerne la seconde, précisait-il, « la délivrance de l'autorisation préalable aux individus exerçant certaines professions ambulantes une fois effectuée par le Préfet du domicile, ou, pour les étrangers, par le Préfet du département frontière, les permissionnaires ont le droit d'exercer leur industrie sur tout le territoire avec l'assentiment des maires $»^{12}$ ), il a fallu que le préfet rectifie alors les dispositions prévues à l'article 2 de son arrêté.

7 Aux deux Chambres, élus et membres du gouvernement, quelle que soit leur appartenance politique, à l'exception des rangs de l'extrême gauche, s'accordent par la suite sur la nécessité de travailler à l'élaboration d'un texte de loi susceptible de mieux contrôler et surveiller les Tsiganes. En témoigne, lors de la séance du 29 octobre 1907, l'intervention de Fernand David, député républicain de Haute-Savoie, qui s'en prend avec véhémence aux nomades et se montre particulièrement suspicieux envers les " romanichels » étrangers (à l'occasion d'un incident survenu à la frontière suisse où des nomades avaient été refoulés vers la France), persuadé que ceux-ci, reconnaissables à " un signe de race", sont des gens dangereux, ignorants, sans culture, et contre lesquels il souhaite que le gouvernement prenne des mesures de police beaucoup plus énergiques : «Il y a deux sortes de nomades : les étrangers et les Français. Les étrangers me préoccupent plus particulièrement, parce que ce sont surtout ceux qui dévastent les régions de l'Est et aussi celles du Midi [...]. Il y a certains Français, qu'on appelle des « roulottiers » et dont s'occupent beaucoup nos collègues de ces départements. Ils sont souvent aussi malfaisants que les nomades étrangers. Pourquoi poursuivons-nous ces gens-là ? Parce qu'ils n'ont pas de domicile, pas d'état civil, parce qu'ils ne travaillent pas (Applaudissements sur un grand nombre de bancs), parce qu'ils vivent de vols et de rapines, et j'attends qu'il se lève ici quelqu'un pour les défendre. (Nouveaux applaudissements sur les mêmes bancs. - Interruptions à l'extrême gauche)"; "Quand un nomade étranger est entre les mains du juge d'instruction, si on lui demande où sont ses enfants, où il s'est marié, où il a enterré ses morts, il répond qu'il a enterré les siens sur la voie publique ou quelque part dans un champ ; que ses enfants sont nés dans sa roulotte, dans une localité dont il a oublié le nom; et qu'en ce qui concerne son pays, il est venu au monde quelque part, lui aussi, dans un lieu inconnu dont il n'a pas conservé le souvenir $»^{13}$. Le vœu du député sera en partie exaucé l'année suivante. Entre-temps, le marquis Robert de Pomereu, député de la $2^{\mathrm{e}}$ circonscription de Rouen, inscrit au 
groupe d'action libérale qui siège à droite de la Chambre, membre de la Société des agriculteurs (cf. J. Jolly, 1960-1977, t. VII : 2726), dépose lui aussi, en date du 20 décembre 1907, une proposition de loi «relative aux moyens propres à réprimer le vagabondage et la mendicité exercés par les nomades étrangers ", précédée d'un long exposé des motifs où les Tsiganes sont assimilés à des «professionnels du crime et du vol », proposition reprenant à son compte l'idée préalablement exprimée de l'obligation d'une double autorisation préfectorale (du département frontière) et municipale imposée aux nomades étrangers. De son côté, le député Étienne Flandin (Union républicaine), qui deviendra sénateur en 1909 et prendra une part importante, avec le député du Doubs Marc Réville (Républicains radicaux et démocratiques), dans l'élaboration de la future loi, entend durcir et étendre les mesures de surveillance à l'égard de tous les nomades, français ou étrangers, comme l'atteste l'article 8 de son texte :

«Tous nomades qui, n'ayant ni domicile ni résidence fixe, voudront exercer une profession ambulante seront tenus de se munir d'une autorisation écrite du préfet du département dans lequel ils entendront circuler.

Tous nomades arrivant dans une commune devront présenter cette autorisation au maire avec leurs feuilles signalétiques visées par la préfecture. Les maires viseront ces feuilles à l'arrivée et au départ.

Toute contravention aux dispositions du présent article constituera les nomades contrevenants en état de vagabondage et les rendra passibles des peines édictées par le Code pénal $»^{14}$.

8 Avec le projet de loi présenté le 25 novembre 1908 au nom du gouvernement Fallières par le ministre de l'Intérieur Georges Clemenceau, une étape décisive est franchie, non seulement parce que les dispositions prises à l'égard des Tsiganes s'inscrivent au cœur de la législation à venir sur la réglementation des professions ambulantes et la circulation des nomades, le nomadisme bohémien ne relevant plus ainsi, du moins en apparence, de la question du vagabondage, mais à cause de la sévérité des formes spécifiques du contrôle administratif et policier prévu par ce projet qui préfigure la loi de 1912. Rappelons au passage que, sous ce même ministre Clemenceau, l'administration française avait commencé par adopter des procédés nouveaux pour assurer une surveillance plus étroite des nomades. Une circulaire de l'Intérieur du 4 avril 1908, qui prescrivait aux brigades mobiles de police de photographier « chaque fois qu'ils en auront la possibilité, les vagabonds, nomades et Romanichels, circulant isolément ou voyageant en groupe ", allait être précédée moins d'un an auparavant d'opérations diverses montées par la Sûreté générale et relayées par la presse. Opérations destinées à convaincre les parlementaires de la nécessité de créer un service actif de police judiciaire, constitué par ces «Brigades régionales de police mobile » (J.M. Berlière, 2000: 311-323). L'action la plus spectaculaire fut organisée contre une troupe de cent nomades, commandée par un certain «Jean Capello ». Partie des PaysBas, elle arriva en France en août 1906; parfois disséminée en petits groupes, elle devait se concentrer en Charente-Inférieure pour la grande foire ( «la frairie ») de La Tremblade, le 2 juin 1907. Ce fut là qu'eut lieu le vaste coup de filet, décidé par Hennion, directeur de la Sûreté générale. Les perquisitions dans les roulottes ne donnèrent cependant qu'un maigre résultat : des jeux, quelques papiers, trois livrets de caisse d'épargne. Cinquante nomades furent arrêtées et interrogés; dix-sept furent relâchés. Le lendemain, arrivèrent, avec leur matériel, les fonctionnaires du service anthropométrique de la Seine. Conformément au "système Bertillon " ${ }^{15}$, les nomades furent photographiés, et l'on prit leurs mensurations et leurs empreintes digitales. 
Dans le contexte des discours sécuritaires et xénophobes qui prévalent à cette époque, où l'intégration nationale des citoyens s'accompagne d'une unification relative des perceptions de l'étranger et son rejet (L. Dornel, 2004 : 225-311), les représentations véhiculées par la société sur la communauté tsigane obéissent aux normes de l'idéologie dominante, laquelle s'inspire aussi en ce domaine des croyances et jugements négatifs hérités comme effet de mémoire de la longue durée (E. Filhol, 1998, 2006). D'où les stéréotypes les plus étroits, les images caricaturales, un catalogue d'idées reçues appliquées aux Tsiganes pour les dévaloriser et ainsi justifier leur rejet

Le projet de loi du gouvernement repose sur un principe de classement introduit entre plusieurs catégories d'itinérants, selon le critère de distinction lié à une condition de domiciliation : l'existence ou non d'un domicile fixe. Cette notion juridique induit deux statuts différents: d'une part les ambulants qui, dotés d'un domicile fixe, ne sont astreints qu'à une simple déclaration, en échange de laquelle ils reçoivent un récépissé qu'ils doivent présenter à toute réquisition; et d'autre part les nomades qui, en étant privés, doivent solliciter une autorisation administrative délivrée sous la forme d'un carnet spécial, dit «carnet d'identité $»^{16}$. D'après les considérations préliminaires contenues dans le projet, la notion de «nomade » désigne en premier lieu les forains, relativement faciles à surveiller, ou à rechercher, lorsqu'ils sont amenés à commettre des tromperies et "vraisemblablement beaucoup de maraude", individus souvent porteurs de pièces d'identité, exerçant leur industrie aux centres des communes qui les y autorisent; ensuite les «roulottiers ou romanichels", définis négativement comme "sans état civil, sans domicile fixe", accusés de se livrer au braconnage et à la mendicité, dont les métiers ne sont aux yeux des pouvoirs publics qu'un alibi pour ces activités. La définition des forains par leurs professions pourrait aussi bien s'appliquer aux personnes et familles taxées de romanichels, car ils exercent les métiers de ceux que la presse qualifie souvent de saltimbanques: artistes ambulants, tenanciers de chevaux de bois, acrobates, chanteurs et musiciens, montreurs d'animaux, somnambules, tireuses de cartes. Il n'est donc pas fait une séparation bien nette entre les uns et les autres, hormis l'hostilité plus grande à l'égard de ceux qui, « exploitant et rançonnant les populations, et troublant parfois par de monstrueux attentats, la tranquillité des campagnes ", renvoient à la désignation vague d'un groupe appelé « romanichel ». Distinction d'ailleurs inutile au regard du critère retenu, l'absence de domicile fixe. Contre les Tsiganes (comme les forains non-tsiganes), le gouvernement prescrit un système de surveillance rigoureux, puisque le carnet d'identité devient obligatoire pour tout "nomade", sous peine d'un mois à un an d'emprisonnement. Le projet de loi établit, en outre, un carnet collectif. Il impose aussi une formalité administrative au propriétaire qui concède aux nomades gratuitement ou à un prix d'argent le droit de stationner sur son terrain : d'après l'article 15 le propriétaire qui se trouve dans ce cas doit dans les 24 heures, avertir le maire de la présence des nomades sur son terrain et cela sous peine d'amende. En ce qui concerne le chapitre des sanctions, on notera que toutes les peines encourues (liées à la fabrication ou falsification d'un carnet, à l'inscription d'un faux nom, au refus de présentation du carnet, à l'absence de visas des autorités qui auraient dû y être apposés, ou encore à son mauvais état, en raison de feuilles déchirées ou maculées) figureront au carnet d'identité et qu'à chaque fois où il $\mathrm{y}$ aura une infraction aux règlements commise par un nomade étranger, les voitures et animaux pourront être provisoirement retenus, à moins de caution suffisante. Autre façon de préconiser une sédentarisation forcée des Tsiganes, en empêchant les familles de voyager. Le gouvernement prévoyait en même 
temps d'instituer un fichier central, dont la tenue incomberait au service du contrôle des recherches judiciaires relevant de la Sûreté générale (ministère de l'Intérieur), un moyen technique efficace mis à sa disposition pour vérifier avec soin l'identité des nomades, de manière à ce que, comme le réclamait déjà dans son rapport conclusif la commission de Marcère en 1898, "de degré en degré, jusqu'au sommet, jusqu'au ministre de l'intérieur, la police redevienne ce qu'elle doit être, une affaire d'État ».

Sur ces entrefaites, la législature 1906-1910 prit fin. L'essentiel du dispositif, auquel fut substituée l'idée (du député Réville, dans le texte de la commission présenté le 7 juin 1909) de l'imposition d'un "carnet anthropométrique d'identité $~^{17}$ plutôt que d'un carnet d'identité pour les nomades qui circulent en France, est toutefois repris par la nouvelle Chambre lorsque celle-ci le soumet au Parlement. À ceci près qu'une modification importante touchant le statut des nomades y est apportée. Le projet va en effet distinguer nettement deux types de nomades: la catégorie des «forains" de nationalité française et celle des "nomades " proprement dits, à savoir les Tsiganes, désignés sous le terme de bohémiens ou romanichels. Pourquoi une telle distinction at-elle été ajoutée ? Parce que les forains, dont le poids électoral n'est pas négligeable auprès des élus, ont protesté au nom de l'égalité pour tous contre des dispositions qui tendent à les assimiler à une population criminelle, qu'ils ne voulaient pas être confondus avec les "romanichels", et ont refusé le "bertillonage " qu'entend instaurer une loi d'exception. La résistance des forains est d'ailleurs soutenue par la presse et bien vite les sénateurs donnent droit à leur revendication. Si bien que la commission sénatoriale sépare les mesures applicables aux forains, en exigeant d'eux la production d'une carte d'identité mentionnant leur signalement accompagné d'une photographie, tandis qu'elle astreint durement les nomades à l'obligation de présenter un carnet anthropométrique d'identité, et à faire viser leur carnet dans les localités où ils se rendent. Et ce n'est pas uniquement le critère de la nationalité française qui se trouve ici pris en compte, puisque la définition des "nomades", regroupant les itinérants dépourvus de domicile fixe, précise " quelle que soit leur nationalité ». Cette catégorisation discriminatoire et xénophobe n'englobe donc pas que les Tsiganes étrangers (et les forains de nationalité étrangère considérés comme nomades) mais également les nomades (Tsiganes) français. C'est dire combien les Tsiganes vivant en France se voient privés des droits inhérents à la citoyenneté nationale, et tombent sous le coup d'une violence arbitraire déployée par l'État, violence tout à la fois politique et juridique, au prétexte supplémentaire que, selon l'idéologie dominante, ces nomades à l'évidence non seulement "prétendent exercer un métier " mais sont "généralement des étrangers ${ }^{18}$.

11 Le projet ainsi remanié, avec quelques autres révisions, fut présenté au Sénat et adopté le 12 mars 1912. Il revint alors devant la Chambre des députés qui l'accepta sans modification dans la séance du 20 juin 1912. La loi, promulguée le 16 juillet 1912, parut au Journal Officiel le 19 du même mois.

\section{Le carnet anthropométrique d'identité}

12 La loi sur l'exercice des professions ambulantes et la réglementation de la circulation des nomades oblige tout nomade, quelle que soit sa nationalité, à faire viser (avec l'indication du lieu, du jour et de l'heure) son carnet individuel établi dès l'âge de 13 ans révolus, à l'arrivée et au départ de chaque commune, comme elle le contraint en 
vertu du décret du 16 février 1913 à se soumettre aux différentes mensurations et identifications photographiques (une double photographie, de profil droit et de face) consignées sur ces carnets : «la hauteur de la taille, celle du buste, l'envergure, la longueur et la largeur de la tête, le diamètre bizygomatique [largeur du visage], la longueur de l'oreille droite, la longueur des doigts médius et auriculaires gauches, celles de la coudée gauche, celle du pied gauche, la couleur des yeux, les empreintes digitales et les deux photographies du porteur du carnet $»^{19}$. Pour ce qui est de l'établissement du carnet anthropométrique, semblable dans son format à un livret militaire et contenant au début 208 pages, le ministère de l'Intérieur a prévu que ces mensurations soient accomplies par les commissaires ou les inspecteurs des brigades mobiles, à défaut, par les agents des services anthropométriques qui ont été créés dans plusieurs villes. Dans le cas où le personnel ne serait pas disponible, il est nécessaire de faire appel aux gardiens des prisons, mais il faut que les nomades soient accompagnés individuellement par des gendarmes ou des policiers. Pour le ministre, les nomades sont potentiellement si dangereux qu'il convient d'éviter, dans un but sécuritaire, de les laisser seuls avec un agent ou de les amener à plusieurs dans les prisons ${ }^{20}$. La loi prévoit par ailleurs que les nomades circulant sans carnet seront considérés comme des vagabonds, et à ce titre soumis aux peines encourues pour ce délit. En outre, le carnet du nomade, où se trouve indiqué son état civil, comporte une partie réservée aux mesures sanitaires et prophylactiques auxquelles les nomades sont assujettis. Qui dit contact avec ces familles dit risque de contamination. Par rapport aux préoccupations hygiénistes de l'époque, au statut métaphorique dominant, symbolisé par la figure exemplaire de Pasteur, où l'éradication des maladies, microbes et épidémies est le corollaire du progrès, tous ceux qui menacent la santé du corps social passent pour être des foyers infectieux dont il faut par conséquent se prémunir.

De nouvelles instructions prises en 1926, il importe de le souligner, entraîneront des dispositions spécifiques à l'égard des nomades de nationalité étrangère, qui auront l'obligation d'acquitter une taxe pour la délivrance et le renouvellement du livret et de faire remplacer celui-ci dans les deux ans ${ }^{21}$. Mais la législation dans sa volonté de contrôle ne se limite pas seulement à l'imposition du carnet anthropométrique d'identité. Les nomades voyageant en "bandes ", c'est-à-dire en groupe ou en famille, doivent aussi se munir d'un carnet collectif. Le chef de famille, porteur de ce carnet, en est le responsable légal. La première page du document le concerne. Les indications qui s'y trouvent sont comparables à celles contenues dans le carnet individuel, à ceci près que la partie "signalement» est moins fournie. En plus du cadre consacré aux "marques particulières", il y a une rubrique pour les autres personnes qui renferme divers renseignements sur l'état civil des individus concernés. Chaque modification apportée à la constitution de la famille doit être inscrite dans cette rubrique et être visée par un officier de l'état civil. À la deuxième page, les liens qui rattachent les membres du groupe au « chef de famille » devront y figurer. Ils peuvent être familiaux, professionnels ou autres. Ils sont consignés en premier; ensuite, c'est l'état civil de la personne qui constitue ce lien, ainsi que son « signalement », qui sont enregistrés. Un encadrement supplémentaire est ajouté pour y recueillir les empreintes digitales des enfants de moins de treize ans ${ }^{22}$. Tous les actes de naissance, de mariage ou de décès qui interviendront ultérieurement devront être mentionnés sur le carnet collectif. De même, lorsqu'un nouveau membre s'adjoint ou quitte le groupe. 

photographies pour les enfants de cinq à treize ans ${ }^{24}$, soit une notice collective, conservées par les préfectures et les sous-préfectures, qui les rangent dans des fichiers dont un exemplaire, faisant l'objet d'un classement centralisé, est envoyé à la Direction de la Sûreté générale du Ministère de l'Intérieur. Sans oublier, en application de la circulaire du 12 octobre 1920, la création d'un "registre à feuillets mobiles », véritable répertoire alphabétique recueillant les noms et numéros des carnets, les lieux de provenance et de destination, la date et l'heure du passage des (ou du) nomades. Sa tenue incombe à la même autorité qui vise les carnets anthropométriques: commissaire de police, commandant de brigade de gendarmerie, ou les maires. Une telle mesure cherche à combler d'après les directives de l'Intérieur un certain laxisme car « jusqu'à présent les autorités qui visaient les carnets de nomades ne prenaient pas note du stationnement ou du passage de ces individus $»^{25}$. Les feuillets mobiles sont mis en place pour pallier cette insuffisance et sont destinés « à faciliter la découverte des nomades recherchés pour crimes et délits ». 
Document : couverture, signalement et visas d'un carnet anthropométrique établi en 1936
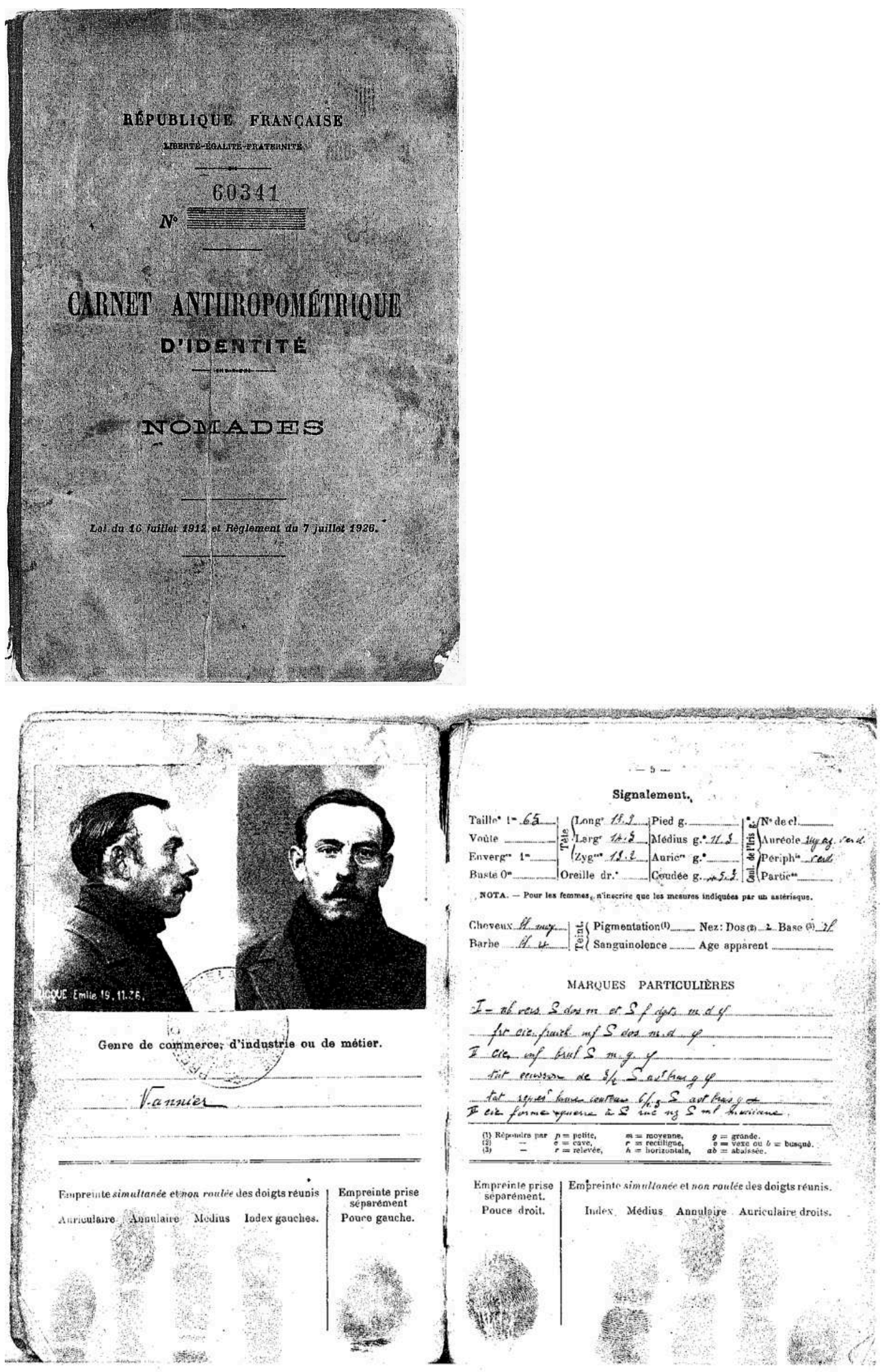


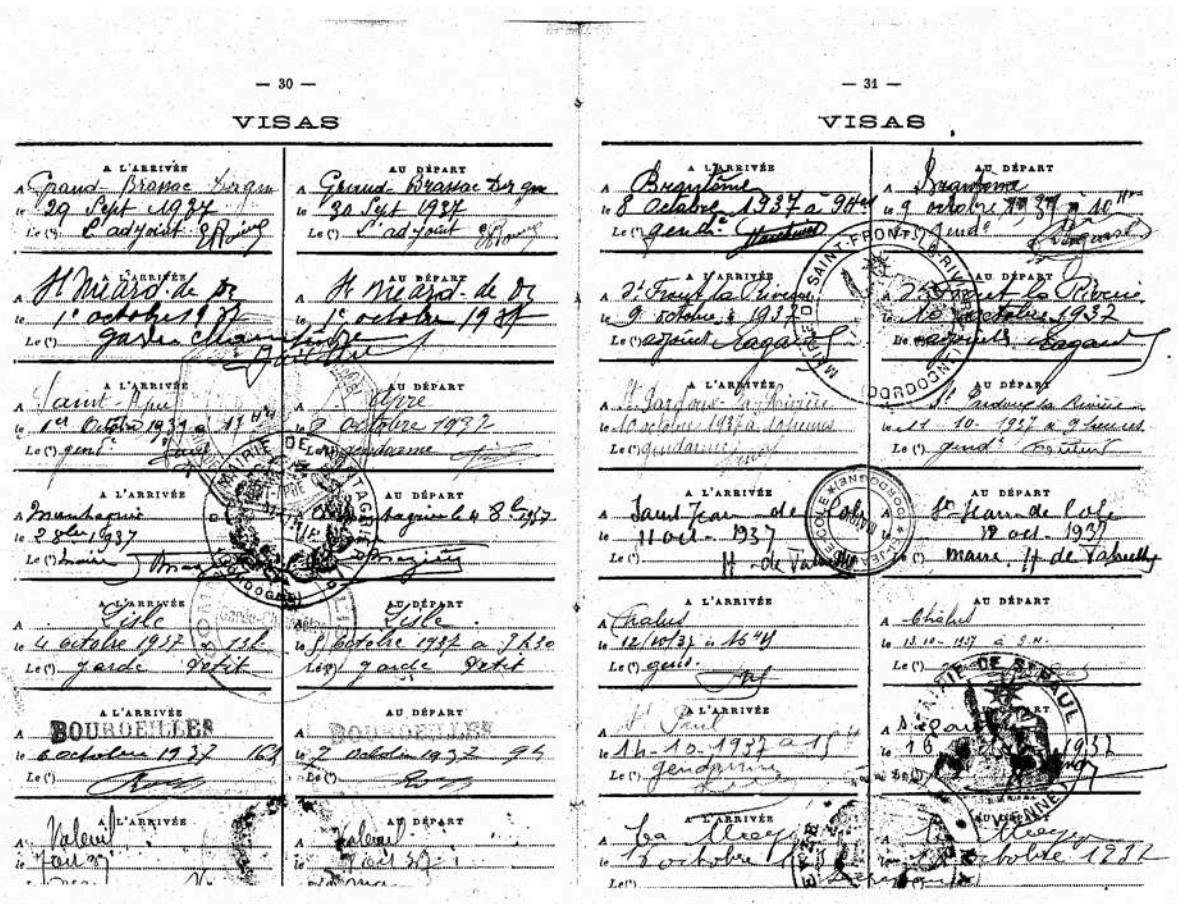

Le recours à différentes pratiques de rationalisation identificatrice de plus en plus contraignantes permet de comprendre la logique d'exclusion prise par la République envers les nomades. Cela va de la simple déclaration à l'«encartement " anthropométrique. Ce procédé vise à assimiler des individus itinérants à une population perçue et construite comme délinquante, criminelle, qu'il s'agit donc d'identifier et de contrôler ${ }^{26}$, dans le but clairement avoué « d'obliger les nomades à se fixer $\aleph^{27}$.

\section{Les commentaires de la loi}

Si l'on en juge d'après les commentaires des juristes, la loi de 1912 n'a pratiquement suscité lorsqu'elle fut rendue publique aucune objection ni discussion critique dans le champ des études juridiques. Le texte sur la circulation des nomades s'est imposé sans réserve auprès des spécialistes du droit comme une mesure d'évidence tout à fait justifiée et bénéfique. Ce qu'atteste en particulier le consensus quasi unanime partagé par les auteurs de travaux universitaires qui lui ont été consacrés. Ainsi Félix Challier (1913 : 446) estime que la promulgation de cette loi répressive, dont il semble illusoire d'attendre un résultat immédiat, servira surtout aux générations suivantes, à condition que législateurs et administrateurs soumettent les nomades à une surveillance et un contrôle incessants. S'inspirant des paroles prononcées par le député Réville, selon lesquelles une durée de vingt ans d'application de la loi serait nécessaire pour « venir à bout du vagabondage en bande ", il ajoute : "Qu'est-ce que vingt ans, en effet, et, si les fils des paysans qui cultivent aujourd'hui le sol de France devaient ne plus connaître, à travers leurs campagnes, les pérégrinations des éternels errants, le législateur n'auraitil pas accompli une œuvre merveilleuse !». La question bohémienne aurait cependant mérité un traitement encore plus rigoureux, en interdisant strictement l'entrée de tout nomade en France: «Avant d'établir une réglementation dont le but détourné, mais certain, est d'obliger les errants à quitter la France, n'était-il pas élémentaire de ne 
point y laisser pénétrer ceux qui ne s'y trouvaient pas encore ?» (Challier, ibid. : 447). Et pourquoi ne pas imaginer un jour, à la suite de ce régime nouveau appliqué aux nomades, qu'une entente entre différents États aboutisse à la décision de «repousser en Asie tous ces nomades et leur interdire l'accès des grands États européens » (G. Torlet, 1913 : 101-102). Dans sa thèse soutenue en 1914, Girard de Coëhorn (1914 : 198) se réjouit des dispositions adoptées envers les nomades, car la loi lui paraît d'une utilité très grande : «Rigoureusement appliquée, elle permettra de suivre les nomades, par conséquent de réprimer leurs crimes et délits. [...]. La loi les rendra moins dangereux. Ce résultat suffirait pour que cette loi soit très appréciée, spécialement des populations des campagnes, qui ont tant à souffrir des Romanichels, et que l'on ne saurait jamais trop protéger ». Ces romanichels n'apparaissent pourtant pas, ou si peu, au cours de la même année, en tant que dangereux malfaiteurs, dans le Bulletin hebdomadaire de la police criminelle, diffusé aux procureurs généraux, juges d'instruction, commissaires de police, commandants de gendarmerie, directeurs de maisons centrales, gardiens-chefs de maisons d'arrêt, afin de retrouver les personnes inculpées de crimes et délits, puisque, sur 4232 personnes recherchées, seuls 7 nomades sont concernés ${ }^{28}$ ! Encore le sont-ils pour de menus larcins. N'importe, un discours identique prévaut chez Henri Arsac (1933 : 461), qui approuve du même coup sans hésitation la circulaire du ministre de l'Intérieur Pierre Laval du 24 août 1931 au sujet de la surveillance des nomades étrangers, dont l'intention louable est d'« interdire à ces indésirables l'accès de notre pays », ou chez Henri Soulé-Limendoux (1935 : 186), assez satisfait des résultats obtenus depuis l'application de la loi, mais soucieux de voir régler le problème des nomades étrangers grâce à une convention susceptible «de débarrasser à tout jamais le territoire national de ces éternels vagabonds ». Évoquons enfin ce que dit, en 1950, Marcel Waline (cité par E. Aubin, 1996: 37-38) : l'éminent professeur de droit public présente la loi comme « un cas probablement unique dans le droit français [...] de législation appliquée à une certaine catégorie de gens, les nomades, un régime d'exception, rejetant cette catégorie hors du droit commun", mais légitime quand même cette législation à l'encontre des bohémiens qui sont, affirme-t-il, "une cause spéciale de péril pour la sécurité et la salubrité publiques ».

19 La loi vue par la presse et l'administration juridiques est l'objet d'appréciations tout aussi favorables. D'aucuns s'accordent à reconnaître que cette « législation nouvelle s'imposait pour assurer la sécurité des campagnes et empêcher les criminels et les voleurs de se joindre aux nomades pour dépister les recherches de la police $»^{29}$. Le long compte rendu paru dans la Revue de Législation et de Jurisprudence vise à expliquer le bien-fondé de la loi, au nom de la sécurité et du bon ordre de la société ; l'avocat Paul Dupont (1913: 124) se félicite entre autres que, parmi les dispositions générales, le législateur ait veillé à centraliser les fiches des nomades : "La centralisation de toutes les fiches signalétiques au ministère de l'Intérieur est une bonne précaution; en cas de crime ou de délit commis par un romanichel, son identification se fera très facilement ». Quant au Conseil d'État, consulté sur le projet de décret qui lui est soumis le 7 novembre 1912 par le ministre de l'Intérieur Steeg, sa seule réserve, de pure forme, semble concerner l'aspect définitionnel sous lequel les nomades apparaissent à l'article $7 \mathrm{du}$ projet de décret, puisque la version initiale, telle que l'avait rédigée le 2e Bureau de la Sûreté générale («Les nomades auxquels l'article 3 de la loi du 16 juillet 1912 impose l'obligation de se munir du carnet anthropométrique d'identité sont: 1e Les individus de nationalité française circulant en France sans domicile ni résidence fixe et ne rentrant pas dans les catégories des ambulants et des forains, même s'ils ont des 
ressources ou prétendent exercer une profession; 2e Les personnes de nationalité étrangère qui n'ayant en France ni domicile ni résidence fixe veulent exercer en France la profession de commerçants ou industriels forains»), sera remplacée par l'énoncé suivant: « Tout individu réputé nomade dans les conditions prévues à l'article 3 de la loi du 16 juillet $1912 \ldots »^{30}$. Comme si, la «nationalité française », relative aux Tsiganes, ne pouvait leur être reconnue, attendu qu'ils sont "presque toujours étrangers", et que la qualité de français, revêtant pour le moins, dans le contexte du discours, une marque identitaire valorisante, s'avérait contradictoire avec leur statut de nomades. Sans doute eût-il été gênant, de surcroît, au regard du droit commun, d'associer explicitement une mesure aussi discriminatoire que le carnet anthropométrique à une partie de la population «française». Il convenait donc plutôt, en vertu de la formulation plus vague et imprécise, à connotation négative, prévue par la loi, de réprimer les nomades, "quelle que soit leur nationalité ». Lors de la séance de la Société générale des Prisons du 17 juin 1914 consacrée à la loi sur les nomades, le caractère " anormal » d'une pareille mesure n'échappa d'ailleurs pas à M. Hennequin, directeur honoraire au ministère de l'Intérieur, qui la justifia cependant, « étant donné que les roulottiers constituent une catégorie de gens éminemment suspects, que l'intérêt public exige qu'ils disparaissent, l'application de l'anthropométrie s'explique parfaitement $»^{31}$.

Comparée aux législations des autres pays européens sur les Tsiganes, la réglementation française de 1912 est la seule à avoir imposé aux nomades le système disciplinaire du carnet anthropométrique. Les pays voisins ne sont pas allés jusque-là, ou l'ont fait à un degré moindre, de façon incomplète, même si leur politique en matière d'identification vis-à-vis des Tsiganes les ont conduits à instaurer diverses pratiques de surveillance et de contrôle très sévères matérialisés par des permis de circulation ou des "papiers d'identité ». Dans les États germaniques, la Bavière, après avoir recensé les Tsiganes en 1905, la publication du livre intitulé Zigeunerbuch, un document d'Alfred Dillmann, chef de la police de Munich, traduisant le résultat des données récoltées (dont une série de photographies), procéda à partir de 1911 à la prise des empreintes digitales de tous les Tsiganes voyageurs. En 1926, l'assemblée législative bavaroise promulgua une loi pour combattre les Tsiganes. Selon la nouvelle loi, ceux qui voulaient voyager avec des roulottes et des caravanes devaient obtenir un permis de police. Ce permis n'était valable que pour une période d'un an et pouvait être retiré à tout moment. Il était interdit de voyager avec des enfants, sauf lorsque les dispositions adéquates pour leur éducation avaient été suivies. Il fallait une autorisation de la police pour emmener des chevaux, des chiens et autres animaux à des fins commerciales. Il était interdit de voyager " en hordes ", une horde étant tout groupe d'individus ou plusieurs familles. Les autorités pouvaient imposer des itinéraires spéciaux aux nomades, elles étaient susceptibles de leur interdire de séjourner dans certaines localités ou leur assigner un lieu de résidence particulier. Les Tsiganes étrangers étaient soumis à ces mesures même en l'absence d'un casier judiciaire. Stimulé par la Bavière, le ministère prussien de l'Intérieur décréta en novembre 1927 au nom de la " lutte contre la nuisance tsigane " le recours obligatoire à la dactyloscopie pour tous les Tsiganes non sédentaires âgés de plus de six ans. Les gouvernements non prussiens furent pressés d'adopter la même mesure, et la plupart des États allemands le firent effectivement ${ }^{32}$. En Autriche, un projet de loi, dit « loi des Tsiganes ", vit le jour en 1931. Il prescrivait que, sous peine de condamnation, les Tsiganes devaient se faire dactyloscopier et photographier. Dès l'âge de six ans, tout 
nomade recevrait une "carte de légitimation spéciale » reproduisant sa photographie et ses empreintes digitales. Il se verrait dans l'obligation de la porter continuellement avec lui. Le projet conditionnait aussi la circulation des familles à l'obtention d'une autorisation pour pouvoir voyager, tandis que celle-ci indiquerait les chemins et territoires pour lesquels elle serait valable. L'autorisation de campement pour la nuit restant soumise à l'appréciation du bourgmestre ou de l'autorité policière intéressée. En ce qui concerne les enfants en âge de scolarité, le texte prévoyait ni plus ni moins d'enlever aux Tsiganes tous les enfants dont l'éducation serait soi-disant en danger et de les placer, si possible, dans des maisons d'éducation spéciale ${ }^{33}$. Une politique qui s'inspirait visiblement de ce qui avait déjà cours en Suisse, où l'ÆEuvre d'entraide pour les enfants de la grand-route créée en 1926 par Alfred Siegfried traquait les enfants tsiganes en bénéficiant de la collaboration sans faille de la police et des autorités ${ }^{34}$. Mentionnons encore, parmi les mesures appliquées en Europe, le traitement administratif que la Belgique réserva en 1933 aux « romanichels », quand la Police des Étrangers décida d'établir leur identité, les soumit à photographie et dactyloscopie et leur délivra une feuille de route munie de photo et valable trois mois. En décembre 1941, à l'initiative de la Police des Étrangers fut instaurée en remplacement de la "feuille de route " la "carte de nomade ", laquelle devint obligatoire, à partir de janvier 1942, pour tout nomade âgé de 15 ans révolus. Tenant lieu de « permis de séjour provisoire ", la carte de nomade - dénommée significativement en néerlandais zigeunerkaart - avait une validité de trois mois. Mais le 5 de chaque mois, elle devait être visée par le commandant de la brigade de gendarmerie la plus proche du lieu de séjour ${ }^{35}$.

La loi de 1912, qui paradoxalement contribua à souder l'identité collective bohémienne en obligeant les familles à voyager ensemble (H. Asséo, $220: 311$ ), eut indirectement des incidences graves et dramatiques sur le sort des Tsiganes en France. Durant la Grande Guerre, les Tsiganes alsaciens-lorrains dotés de carnets anthropométriques, ceux qui avaient opté pour la France après la capitulation de 1871, furent arrêtés et dirigés avec d'autres nomades évacués de la zone du front vers les dépôts surveillés de l'Ouest et du Midi. L'internement des "romanichels" dura toute la guerre, et au-delà (E. Filhol, 2004). Vingt ans plus tard, ou presque, la République finissante décréta le 6 avril 1940 l'assignation à résidence de tous les "nomades » définis selon l'article 3 de la loi de 1912, au motif que leur circulation constituait "pour la défense nationale et la sauvegarde du secret un danger qui doit être écarté ». Une partie de ceux-ci, environ cinq mille personnes, hommes, femmes et enfants, à la suite d'une décision de l'occupant allemand, aboutira en zone nord dans des camps gérés par Vichy (D. Peschanski, 1994 ; E. Filhol, 2004) ${ }^{36}$. Certains d'entre eux, déportés de Poitiers, mourront en Allemagne (E. Filhol, 2000). On pouvait escompter, au lendemain de la Libération, que la persécution des Tsiganes aurait incité l'État français à mener une politique plus compréhensive à leur égard. Il n'en a rien été. Les derniers Tsiganes internés seront libérés du camp d'Angoulême en mai 1946, tandis que la loi de 1912 restera longtemps encore maintenue. Ce régime conçu comme un instrument d'ostracisme à caractère racialiste n'allait être abrogé qu'en $1969^{37}$, le « livret ou carnet de circulation », toujours en vigueur, visés chaque mois puis trimestriellement par un commissaire de police ou un commandant de gendarmerie, se substituant alors au carnet anthropométrique. 


\section{BIBLIOGRAPHIE}

ABOUT Ilsen (2004) Les fondements d'un système national d'identification en France (1893-1914). Anthropométrie, signalements et fichiers, Genèses. Sciences sociales et histoire, 54, pp. 28-52.

ARSAC Henri (1933) La loi du 16 juillet 1912 sur l'exercice des professions ambulantes et la circulation des nomades, Thèse pour le doctorat, Université de Lyon, Faculté de droit, Lyon, Bosc Frères, M. \& L. Riou, $470 \mathrm{p}$.

ASSÉO Henriette (1974) Le traitement administratif des Bohémiens dans la société française du XVII ${ }^{e}$ siècle, in Problèmes socio-culturels en France au XVII siècle, Préface de Robert Mandrou, Paris, Klincksieck, pp. 9-87.

ASSÉO Henriette (1994) Les Tsiganes. Une destinée européenne, Paris, Gallimard, « Découvertes », $160 \mathrm{p}$.

ASSÉO Henriette (2002) La gendarmerie et l'identification des «nomades » (1870-1914), in JeanNoël Luc, sous la dir., Gendarmerie, État et Société au XIX ${ }^{e}$ siècle, Paris, Publications de la Sorbonne, pp. 301-311.

AUBIN Emmanuel (1996) À propos d'un texte de Marcel Waline : « Un problème de sécurité publique : les bohémiens ", Études tsiganes, nouvelle série, volume VII, pp. 37-46.

AUBIN Emmanuel (2001) L'évolution du droit français applicable aux Tsiganes. Les quatre logiques du législateur républicain, Études tsiganes, nouvelle série, volume XV, pp. 26-56.

BATAILLARD Paul (1843-1844) De l'apparition et de la dispersion des Bohémiens en Europe, in Bibliothèque de l'École des Chartes, $3^{\mathrm{e}}$ série, 1, pp. 348-375 et 521-552.

BEAUNE Colette (1990), Texte original et intégral présenté et commenté, Le Journal d'un Bourgeois de Paris, de 1405 à 1449, Paris, Le livre de poche, « Lettres Gothiques », 539 p.

BERLIÈRE Jean-Marc (1996), Le Monde des polices en France XIX ${ }^{e}-X X^{e}$ siècles, Bruxelles, Complexe, $275 \mathrm{p}$.

BERLIÈRE Jean-Marc (2000), La seule police qu'une démocratie puisse avouer ? Retour sur un mythe : les brigades du Tigre, in Serviteurs de l'État : une histoire politique de l'administration française 1880-1945, sous la direction de Marc-Olivier Baruch et Vincent Duclert, Paris, La Découverte, pp. 311-323.

BRUBAKER Rogers (1997), Citoyenneté et nationalité en France et en Allemagne, traduit de l'anglais par Jean-Pierre Bardos, Paris, Belin, 320 p.

CHALLIER Félix (1913), La nouvelle loi sur la circulation des nomades. Loi du 16 juillet 1912, Thèse pour le doctorat, Université de Paris, Faculté de droit, Paris, Librairie de Jurisprudence ancienne et moderne E. Duchemin, 459 p.

DELCLITTE Christophe (1995) La catégorie juridique « nomade » dans la loi de 1912, Hommes \& Migrations, 1188-1189, Juin-Juillet, pp. 23-30.

DORNEL Laurent (2004) La France hostile. Socio-histoire de la xénophobie (1870-1914), Paris, Hachette Littératures, $361 \mathrm{p}$.

DUPONT Paul (1913) La loi du 16 juillet 1912 sur les Nomades, Lois Nouvelles. Revue de Législation et de Jurisprudence, Année 1913, pp. 73-128. 
FILHOL Emmanuel (1998) Le mot Bohémien(s) dans les dictionnaires français (XVII ${ }^{\mathrm{e}}$-XIX ${ }^{\mathrm{e}}$ siècle). À propos des formes de l'énoncé, Lexicographica. Revue internationale de Lexicographie, volume XIV, pp. 177-204.

FILHOL Emmanuel (2000) L’internement et la déportation de Tsiganes français : MérignacPoitiers-Sachsenhausen, 1940-1945, Revue d'histoire de la Shoah. Le monde juif, 170, septembredécembre, pp. 136-182.

FILHOL Emmanuel (2002) Les Tsiganes en Lorraine à l'époque de Jacques Callot, Le Pays Lorrain. Journal de la Société d'Histoire de la Lorraine et du Musée Lorrain, volume XVIII, Novembre-Janvier, pp. 57-61.

FILHOL Emmanuel (2003) dir., Histoires tsiganes. Hommage à François de Vaux de Foletier, Catalogue de l'exposition, Archives départementales de la Charente-Maritime, Conseil général de la Charente-Maritime, La Rochelle, Imprimerie Rochelaise, Le nouvel R, 85 p.

FILHOL Emmanuel (2004) Un camp de concentration français. Les Tsiganes alsaciens-lorrains à Crest 1915-1919, Grenoble, Presses Universitaires de Grenoble, 181 p.

FILHOL Emmanuel (2004) La mémoire et l'oubli : l'internement des Tsiganes en France, 1940-1946, Paris, L'Harmattan, Centre de recherches tsiganes de l'Université Paris V, 119 p.

FILHOL Emmanuel (2006) La Bohémienne dans les dictionnaires français (XVIII Discours, histoire et pratiques socio-culturelles, in La Bohémienne, figure poétique de l'errance aux XVIII et XIX ${ }^{e}$ siècles, sous la direction de Pascale Auraix-Jonchière et Gérard Loubounoux, Clermont-Ferrand, Presses Universitaires Blaise Pascal, pp. 13-35.

FILHOL Emmanuel (en lecture), Le traitement administratif des Bohémiens dans les Landes (1895-1946). Annales du Midi, 20 p. dact.

FRASER Angus (1992), The Gypsies, Oxford, Blackwell, 359 p.

GIRARD DE COËHORN Pierre (1914) Les nomades et la loi pénale, Thèse pour le doctorat juridique, Université de Montpellier, Faculté de droit, Montpellier, Imprimerie Firmin et Montane, 204 p.

GRELLMANN Heinrich M. G. (1787) Historischer Versuch über die Zigeuner, betreffend die Lebensart..., Göttingen, Traduit de l'allemand par M. J., Histoire des bohémiens, ou tableau des moeurs, usages et coutumes de ce peuple nomade, suivie de recherches historiques sur leur origine, leur langage et leur première apparition en Europe, Paris, Joseph Chaumerot, 1810, 356 p.

ISAMBERT, DECRUSY, TAILLANDIER (1821-1833) Recueil général des anciennes lois... depuis l'an 420 jusqu'à la Révolution de 1789, Paris, Belin-Leprieur, tome XIX.

JOLLY Jean, dir. (1960-1977) Dictionnaire des parlementaires français. Notices biographiques sur les ministres, sénateurs et députés français de 1889 à 1940, Paris, PUF.

KALUSZYNSKI Martine (1987) Alphonse Bertillon et l'anthropométrie, in Maintiens de l'ordre et police en France et en Europe au XIX ${ }^{e}$ siècle, sous la direction de Philippe Vigier et al., Paris, Créaphis, pp. 269-295.

LEBLON Bernard (1985) Les Gitans d'Espagne. Le prix de la différence, Paris, PUF, 255 p.

LEIMGRUBER Walter, et al. (2000) L'Oeuvre des enfants de la grand-route, étude historique réalisée à partir des archives de la Fondation Pro Juventute déposées aux Archives fédérales, Office fédéral central des imprimés et du matériel, Berne, $64 \mathrm{p}$.

LEWY Guenter (2003) La persécution des Tsiganes par les nazis, Traduit de l'anglais par Bernard Frumer, Avant-propos par Henriette Asséo (pp. IX-XXIX), Paris, Les Belles-Lettres, 472 p. 
MOSSÉ Armand (1924) Application de la loi du 16 juillet 1912 relative aux marchands ambulants, aux commerçants ou industriels forains et aux nomades, in Journal Officiel, Documents administratifs, 28 septembre, pp. 714-734.

NOIRIEL Gérard (1999) Les origines républicaines de Vichy, Paris, Hachette Littératures, 335 p.

PERNOT Mathieu (2001) textes de Henriette Asséo et Marie-Christine Hubert, Un camp pour les Bohémiens. Mémoires du camp d'internement pour nomades de Saliers, Arles, Actes Sud, 110 p.

PESCHANSKI Denis (1994) avec la collaboration de Marie-Christine Hubert et Emmanuel Philippon, Les Tsiganes en France, 1939-1946, Paris, CNRS Éditions, 176 p.

ROCHE Daniel (2003) Humeurs vagabondes. De la circulation des hommes et de l'utilité des voyages, Paris, Fayard, 1022 p.

SOULÉ-LIMENDOUX Henri (1935) Ambulants, forains et nomades, Thèse pour le doctorat, Université de Toulouse, Faculté de droit, Toulouse, Imprimerie Moderne, $189 \mathrm{p}$.

THURNER Erika (1983) Nationalsozialismus und Zigeuner in Osterreich, Wien-Salzburg, Geyer Edition, $235 \mathrm{p}$.

TORLET G. (1913), Le régime administratif applicable aux nomades et marchands forains, Thèse pour le doctorat, Faculté de droit de Paris, Paris, Imprimerie H. Jardin, 109 p.

VAUX DE FOLETIER François de (1961) Les Tsiganes dans l'Ancienne France, Paris, Société d'Édition Géographique et Touristique, «Connaissance du Monde », 246 p.

VAUX DE FOLETIER François de (1970) Mille ans d'histoire des Tsiganes, Paris, Fayard, 282 p.

\section{NOTES}

1. L'arrivée des premiers Tsiganes, appelés Égyptiens, un groupe composé d'environ cent vingt personnes, sous les ordres d'un certain André qui s'intitule duc de la Petite Égypte, est signalée à Mâçon en 1419 (F. de Vaux de Foletier, 1961 : 18). En août 1427, les Tsiganes apparaissent pour la première fois aux portes de Paris (C. Beaune, $1990: 234-238$ ).

2. À propos de cet âge d'or des Tsiganes, H. Asséo (1994).

3. Sur cet aspect, à la lumière des travaux menés par H. Asséo (en particulier, 1974), voir les remarques de D. Roche (2003 : 947-951).

4. La loi de 1889, comme on sait, fonde la nationalité française sur un double jus soli (selon que la loi impose à l'enfant né en France d'un parent étranger lui-même né en France d'être Français à la naissance tandis que l'enfant né en France de parents étrangers devient français à sa majorité) contrairement à l'Allemagne, qui a toujours conçu la nationalité à partir de la communauté d'ascendance, définie par le système de jus sanguinis pur (R. Brubaker, 1997).

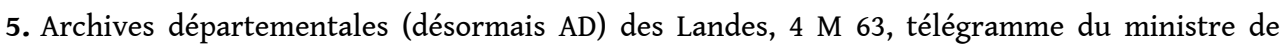
l'Intérieur aux préfets, le 9 avril 1895.

6. Sur le recensement des Tsiganes landais, E. Filhol (en lecture).

7. Le Petit Journal, suppl. illustré du 5 mai 1895, commentaire de la gravure, p. 143.

8. Commission extra-parlementaire sur la police rurale et municipale, rapport du 29 février 1895, par de Marcère, sénateur, Archives nationales, BB18 6442, Circulaires des ministres de l'Intérieur et de la Justice sur la répression du vagabondage et de la mendicité, 1886-1907.

9. Journal Officiel, Rapport présenté au nom de la commission extraparlementaire chargée de rechercher les moyens propres à améliorer la police du vagabondage et des campagnes, par de Marcère, sénateur, 29 mars 1898, p. 1945. 
10. $\mathrm{AD}$ Gironde, $1 \mathrm{~N}$ 126, Procès-verbaux imprimés des délibérations du conseil général, Bordeaux, Imprimerie Delmas, p. 62.

11. Recueil des actes administratifs. Préfecture de la Gironde, novembre 1904, $\mathrm{n}^{\circ} 25$, pp. 385-388 (Instructions et Arrêté).

12. Lettre du Président du Conseil, Ministre de l'Intérieur \& des Cultes à M. le Préfet de la Gironde, Paris, le 12 septembre 1904, AD Gironde, 4 M 246, Instructions, correspondance, notifications de condamnations, rapports sur la mendicité et le vagabondage dans le département..., 1808-1907.

13. Journal Officiel, Chambre des députés. Débats. Séance du 29 octobre 1907, pp. 1973-1974.

14. Journal Officiel, documentation parlementaire, avril 1908, p. 68.

15. Sur le «système Bertillon » de l'« identification anthropométrique » appliquée d'abord aux récidivistes et malfaiteurs à partir de l'année 1883, puis aux nomades et étrangers (M. Kaluszynski, 1987 : 269-295 ; C. Delclitte, 1995 : 28-30 ; J.-M. Berlière, 1996 : 41-68 ; G. Noiriel, 1999 : 188-204 ; I. About, 2004).

16. Journal Officiel, Projet de loi relatif à la réglementation de la circulation des nomades, présenté au nom de M. Fallières Président de la République française, par M. G. Clemenceau, président du conseil, ministre de l'Intérieur. Chambre des députés, Documentation parlementaire, Projets et propositions de loi. Exposés des motifs et rapports, mars 1909, (pp. 111-114), p. 111.

17. Journal Officiel, Rapport supplémentaire de M. Réville, 7 juin 1909. Documents parlementaires, octobre 1909, p. 1213 (article 9).

18. Archives de police de Paris, Boîte DB 200, Rapport de M. Flandin, 30 mars 1911. Documents parlementaires, $\mathrm{n}^{\circ} 101$, (pp. 3-8), p. 3.

19. Bulletin officiel du Ministère de l'Intérieur, 16 février 1913, article 8, p. 79.

20. Instructions du Ministère de l'Intérieur concernant l'application de la loi du 16 juillet 1912, 3 octobre 1913, paragraphe 18.

21. En vertu de l'article 42 de la loi du 4 avril 1926 (cf. Ministère de l'Intérieur, Réglementation de l'exercice des professions ambulantes et de la circulation des nomades, Melun, Imprimerie administrative, 1926, p. 23).

22. Selon les instructions du Ministère de l'Intérieur du 3 octobre 1913, «il n'y a pas lieu de prendre les empreintes digitales des enfants ayant moins de deux ans : c'est seulement lorsqu'ils viennent à dépasser cet âge que le titulaire du carnet collectif doit faire apposer leurs empreintes digitales " (article 18). On trouve cependant, sur certains carnets collectifs, en particulier ceux délivrés en novembre 1913 par la préfecture des Landes, des empreintes digitales d'enfants de moins de deux ans (AD Gironde, 4 M 303, Nomades : carnets collectifs, 1913-1940).

23. AD Gironde, 4 M 303, Ibid.

24. C'est ce que prévoit le décret du 7 juillet 1926, au paragraphe 21 : «En ce qui concerne les enfants de nomades âgés de cinq à treize ans, il est établi [...] une notice individuelle. Cette notice comporte l'état civil, les empreintes digitales des deux mains, la photographie (face et profil) et l'indication, s'il y a lieu, des difformités telles que gibbosité, claudication, perte d'un membre, d'une main, d'un doigt, etc. » (Ministère de l'Intérieur, Réglementation de l'exercice des professions ambulantes et de la circulation des nomades, Melun, op. cit., p. 12). L'identification des enfants sera renforcée par la circulaire du 31 décembre 1928, puisque leurs photos ne devront pas seulement être destinées aux notices individuelles mais collées sur les carnets collectifs: "Une photographie de face et une autre de profil seront collées : $1^{\circ}$ Au verso et en haut du carnet collectif sur lequel figurent l'état civil et le signalement de l'enfant » (AD Gironde, 58 W 82, Nomades et Forains. Instructions 1940-1967).

25. AD Dordogne, 4 M 59, Nomades, forains. Instructions et affaires générales, 1858-1940.

26. A. Mossé, inspecteur général des services administratifs, indique qu'en date du premier septembre 1923 le nombre de nomades dotés de carnets s'élève à 30068 (Journal officiel, 28 septembre $1924: 734)$. 
27. Le mot est de Jules Sébille, contrôleur des recherches au ministère de l'Intérieur, directeur de la police mobile, dans "L'application de la loi sur les nomades ", Séance de la Société générale des Prisons du 17 juin 1914, Revue pénitentiaire et de droit pénal, 6-12, Juin-Décembre 1914, (pp. 805-827), p. 819.

28. Archives nationales, F/7/14619, Bulletin hebdomadaire de la police criminelle, 1914.

29. Lois, Décrets, Ordonnances et Règlements, Nouvelle Série, Tome Douzième, 1913, Paris, Librairie Sirey, Loi sur l'exercice des professions ambulantes et la réglementation de la circulation des nomades, (pp. 408-415), p. 409.

30. Archives nationales, AL 3049, Projet de décret portant règlement d'administration publique pour l'exécution de la loi du 16 juillet 1912 sur l'exercice des professions ambulantes et la circulation des nomades.

31. Cf. Revue pénitentiaire et de droit pénal, article déjà cité, 1914, p. 824.

32. Sur ces aspects, G. Lewy (2003 : 19-25)

33. E. Thurner (1983: 54 et annexe : 1-8, pour le texte du projet de loi).

34. Voir W. Leimgruber, et al. (2000). Continuité discriminatoire oblige : quelques années après la circulaire du 11 juillet 1906 adressée par le Conseil fédéral aux Gouvernements des cantons invitant ces derniers à "s'opposer vigoureusement à l'entrée des Tsiganes sur le territoire ", la Suisse s'était proposée en 1909 d'organiser une conférence internationale (qui finalement n'eut pas lieu) en vue d'un "règlement de la question tsigane" (Documents diplomatiques suisses, Diplomatische Dokumente der Schweiz, tome 5, 1904-1914, Berne, Benteli Verlag, 1983, Protocole... (Protokoll...)/Projet de programme... (Programmentwurf...) du 2 Juillet 1909 [en 17 articles], pp. 583-587).

35. Archives générales du Royaume, Police des Étrangers (2e versement), 1116, Feuilles de route, notes, mesures à prendre, demandes de renseignement et correspondance, 1929-1964.

36. En zone sud, près de sept cents Tsiganes vécurent de 1942 à 1944 dans le camp d'internement de Saliers (Bouches-du-Rhône), créé par le gouvernement de Vichy (M. Pernot, 2001).

37. Les juristes ne manqueront pas de le reconnaître... un peu tard : «Un auteur a qualifié cette loi de raciste car en réalité certaines de ses dispositions visent seulement une catégorie ethnique. La loi fait disparaître les obligations les plus humiliantes qui sont imposées aux nomades, notamment le carnet anthropométrique et à ce titre elle représente un effort de progrès social » (Les Lois Nouvelles. Revue de Législation commentée et annotée, Année 1969, IIe Partie - Lois, Décrets, p. 110). L'argument invoqué par le ministre de l'Intérieur Christian Fouchet consistera à dire, non sans ambiguïté et sous-entendu malveillant, que «le carnet anthropométrique ne présente plus d'utilité réelle dans la recherche des nomades délinquants, alors que ses détenteurs et les personnes qui s'intéressent à leur évolution y voient un procédé de ségrégation difficilement tolérable à l'époque présente " (Journal Officiel, Projet de loi relatif à l'exercice des activités ambulantes et au régime applicable aux personnes circulant sans domicile ni résidence fixe, présenté au nom de M. G. Pompidou, Premier ministre, par M. C. Fouchet, ministre de l'Intérieur. Exposé des motifs, 9 octobre 1968, Documents de l'Assemblée Nationale, Séance du 22 mai 1968, p. 1294). Comme l'a bien montré E. Aubin (2001 : 32), si la loi de 1969 est beaucoup plus libérale que celle de 1912, il reste qu'elle participe d'une logique de la sédentarisation et « qu'elle fait des gens du voyage une catégorie de citoyens français à part puisque ces individus sont les seuls citoyens français dont la liberté d'aller et venir est conditionnée par la possession de titres de circulation ». 


\section{RÉSUMÉS}

À partir de la fin du XIX ${ }^{e}$ siècle, un ensemble de facteurs d'ordre socio-économique, politique, idéologique concourent à assimiler les Tsiganes à des étrangers et des "asociaux indésirables ". La présente contribution tente d'examiner comment, sous la Présidence de la République d'Armand Fallières, la mobilité tsigane a fait l'objet d'un traitement administratif hostile. L'attitude des pouvoirs publics à l'égard des «nomades » français et étrangers s'est déployée en effet autour d'un dispositif législatif et policier que l'on peut résumer ainsi: surveillance, identification, contrôle. Après le recensement général de 1895 de tous les «nomades, bohémiens et vagabonds", suivi du fichage des "nomades" par les Brigades régionales de police mobile créées en 1907 à l'initiative de Clemenceau, un projet de loi du gouvernement daté du 25 novembre 1908 voit le jour, "relatif à la réglementation de la circulation des nomades». Le projet, conjugué avec les mesures émises plus tard à la Chambre et au Sénat par les commissions du vagabondage et de la mendicité, donnera lieu à la loi du 16 juillet 1912 sur le port du carnet anthropométrique d'identité. Cette loi discriminatoire et disciplinaire, qui allait durer près de soixante ans, sans susciter aucune critique au sein du discours juridique dominant, constitue l'étape majeure dans le processus de contrôle et d'identification utilisé par la République envers les Tsiganes.

The Law of 1912 on the Movement of the «Nomads» (Gypsies) in France. From the end of the nineteenth century onwards, a series of socio-economic, political and ideological factors contribute to consider Gypsies as foreigners and "asocial undesirable people". The present contribution aims at explaining how, under Armand Fallières's Presidency, gypsy mobility became the subject of a hostile administrative process. The attitude of the authorities regarding the French and foreign "nomads" was therefore developed through a legal and police device that can be summarized as follows: surveillance, identification, control. After the general census of all the "nomads, Bohemians and vagrants" in 1895, there followed a filing of the "nomads" by the regional Brigades of the mobile police force created in 1907 by Clemenceau, and then a government bill "relating to the regulation of the nomads'movement" emerged on November $25^{\text {th }} 1908$. This project, combined with the measures later emitted in the Chamber of Deputies and the Senate by the Commissions of vagrancy and begging, will lead to the Law of July 16th on the bearing of the anthropometric identification record. This discriminatory and disciplinary law, which was to last for nearly sixty years, without causing any criticism within the prevailing legal discourse, constitutes the major step in the process of control and identification used by the French Republic towards the Gypsies.

La ley de 1912 sobre la circulación de los «nómadas» (gitanos) en Francia. Desde finales del siglo XIX, un conjunto de factores de orden socioeconómico, político e ideológico contribuyeron a asimilar a los gitanos con extranjeros y "asociables indeseables". Esta contribución trata de examinar cómo, bajo la Presidencia de Armand Fallières, la movilidad gitana fue objeto de un trato administrativo hostil. La actitud de los poderes públicos respecto a los "nómadas" franceses y extranjeros se desplegó en torno a un dispositivo legislativo y policial que puede resumirse de la siguiente manera: vigilancia, identificación y control. Tras el censo general de todos los "nómadas, bohemios y vagabundos" de 1895 y el fichaje de los "nómadas" por las Brigadas regionales de policía creadas en 1907 a iniciativa de Clemenceau, el 25 de noviembre de 1908 vio la luz un proyecto de ley del gobierno relativo a la reglamentación de la circulación de los nómadas. Este proyecto y las medidas que tomaron posteriormente las comisiones de vagabundeo y mendicidad de la Cámara y del Senado darán lugar a la ley del 16 de julio de 1912 relativa al porte del carné de identidad antropométrico. Esta ley discriminatoria y disciplinaria 
estará en vigor durante cerca de sesenta años sin suscitar crítica alguna en el seno del discurso jurídico dominante. Se trata de la principal etapa en el proceso de control e identificación de gitanos puesto en práctica por la República.

\section{AUTEUR}

\section{EMMANUEL FILHOL}

Maître de conférences à l'université de Bordeaux 1, Laboratoire Epistémé (EA 2971), Institut

Lamartine, 40, rue Lamartine, 33400 Talence. Email : e.filhol@episteme.u-bordeaux1.fr 